\title{
Pediatric Aortic Valve Repair: Any development in the material for cusp extension valvuloplasty?
}

\author{
Kelli $\mathrm{Hu}^{1}$, Umar Siddiqi ${ }^{1}$, Brian Lee ${ }^{2}$, Emily Pena ${ }^{2}$, Kelci Schulz ${ }^{2}$, Maggie Vogel ${ }^{2}$, Pamela \\ Combs $^{3}$, Chawki El Zein ${ }^{2}$, Michel Ilbawi ${ }^{2}$, Luca Vricella ${ }^{1}$, and Narutoshi Hibino ${ }^{1}$ \\ ${ }^{1}$ The University of Chicago Medicine \\ ${ }^{2}$ Advocate Children's Hospital \\ ${ }^{3}$ University of Chicago
}

August 18, 2021

\begin{abstract}
Background: Aortic cusp extension is a technique for aortic valve (AV) repairs in pediatric patients. The choice of the material used in this procedure may influence the time before reoperation is required. We aimed to assess post-operative and long-term outcomes of patients receiving either pericardial or synthetic repairs.

Methods: We conducted a single center, retrospective study of pediatric patients undergoing aortic cusp extension valvuloplasty $(\mathrm{N}=38)$ with either autologous pericardium $(\mathrm{n}=30)$ or CorMatrix $(\mathrm{n}=8)$ between April 2009 and July 2016. Short and longterm postoperative outcomes were compared between the two groups. Freedom from reoperation was compared using Kaplan Meier analysis. Degree of aortic stenosis (AS) and aortic regurgitation (AR) were recorded at baseline, post-operatively, and at outpatient follow-up.

Results: At five years after repair, freedom from reoperation was significantly lower in the CorMatrix group (12.5\%) compared to the pericardium group $(62.5 \%)(P=0.01)$. For the entire cohort, there was a statistically significant decrease in the peak trans-valvar gradient between pre- and post-operative assessments with no significant change at outpatient follow-up. In the pericardium group, 28 (93\%) had moderate to severe AR at baseline which improved to 11 (37\%) post-operatively and increased to $21(70 \%)$ at time of follow-up. In the biomaterial group, $8(100 \%)$ had moderate to severe AR which improved to 3 (38\%) post-operatively and increased to $7(88 \%)$ at time of follow-up.
\end{abstract}

Conclusion: In terms of durability, the traditional autologous pericardium may outperform the new CorMatrix for AV repairs using the cusp extension method.

\section{Introduction}

Aortic valve repair $(\mathrm{AVr})$ is an interventional strategy used to manage aortic valve disease in children and adolescents. ${ }^{1}$ Among the various choices of techniques for repair, many surgeons favor aortic cusp extension valvuloplasty (ACEV) due to its effectiveness in reducing aortic regurgitation (AR) and aortic stenosis (AS) while improving left ventricular wall mass thickness. ${ }^{2}$ While surgical techniques for repair are well documented in the literature, little clinical evidence exists in determining the optimal material for cusp extension in the pediatric population. ${ }^{3,4}$ When choosing a material for use during repair, durability and functionality must be considered in order to successfully preserve the left ventricle function and minimize the number of reoperations needed over a lifetime. ${ }^{5}$ Currently, the search for the optimal leaflet replacement material requires further exploration.

Aortic valve repair is traditionally accomplished using autologous pericardium, which provides acceptable durability when treated with glutaraldehyde yet suffers from problems with calcification and eventual degradation. ${ }^{4}$ Bovine pericardium is a biological material that was introduced as an alternative to autologous 
pericardium; however, a recent study that compared the two materials demonstrated better performance of autologous pericardium with lower gradient across the aortic valve at final follow-up and less rapid degeneration in children and young adults. ${ }^{5}$ Although identification of leaflet replacement material remains a difficult endeavor, a recently FDA approved synthetic material, CorMatrix (CorMatrix Cardiovascular, Inc, Atlanta, GA), shows promise. Potential benefits include increased durability, resistance to calcification, and propagation of native tissue regeneration. ${ }^{6}$ Due to the novelty of the material, its clinical performance as compared to traditional materials remains undetermined.

To our knowledge, the current study represents the first retrospective comparison of autologous pericardium and CorMatrix material utilized for cusp extension valvuloplasty in pediatric populations. In this study, we aim to identify any predictive factors associated with type of material used for valve repair by assessing short and long-term postoperative outcomes of children and adolescents receiving either traditional autologous pericardium or CorMatrix for AV repair.

\section{Materials and Methods}

Due to this study's retrospective nature, the need for informed consent was waived by our institutional review board (IRB).

\section{Patients}

We performed a retrospective review of patients undergoing aortic cusp extension valvuloplasty at a large, urban academic medical center between April 2009 to July 2016. Patients receiving CorMatrix, either patch or membrane, were compared to those receiving autologous pericardium irrespective of glutaraldehyde time.

\section{Data Collection}

We collected the following baseline characteristics: age, previous operation, aortic valve anatomy, CPB time, and cross clamp time for all patients. Clinical data such as echocardiography and cardiovascular function were reviewed to determine the severity of aortic stenosis (AS) and aortic regurgitation (AR) at baseline, post-operatively, and outpatient follow-up between the two groups.

\section{Statistical Analysis}

Pre-operative and post-operative echocardiography were compared using the 2-tailed paired $t$ test for peak transvalvular gradient across the AV. Statistical significance was set at $\mathrm{P}<0.05$. Overall freedom from reoperation was determine by Kaplan-Meier analysis and is expressed as the percentage of patients who remained event free. If patients had multiple re-operations, the number of days to the first re-operation was used for the analysis. If patients had no listed re-operations, it was assumed that the material was alive at the date of censorship.

\section{Surgical Technique}

ACEV was performed similarly to previously published techniques, and a midline sternotomy was used in all procedures. ${ }^{2,7,8}$ For patients receiving autologous pericardium, a segment of autologous pericardium was harvested and treated with buffered glutaraldehyde solution $(0.625 \%)$. For one patient in 2009, the pericardium was treated with glutalderhyde solution for 10 minutes. However, as our technique evolved in later years, 3 to 2-minute treatment was used, and the pericardium was kept moist with normal saline solution. Further, all CorMatrix patches or membranes were soaked in saline for 10 minutes before implantation per the manufacturer's recommendation.

Following an oblique aortotomy incision on cardiopulmonary bypass (CPB), the extent of tissue deficiency, the shape, and the irregularities of the free edge are evaluated before the thinning of thickened edges of the cusps. The ACEV technique is then performed using the piece of treated and tailored autologous pericardium or CorMatrix, both of which are fashioned to fit each cusp but slightly oversized in depth (10-15\%) and length 
(up to 25\%). The sutures are created using continuous 5-0 or 6-0 polypropylene suture (Prolene; Ethicon Inc, Sommerville, NJ) and are aligned from the cusp's center towards the commissures. ${ }^{7}$

The suture line on the pericardial site is marginally wider than that on the cusp site to provide enough depth to the cusp so that the reconstructed cusp free edge is level with the sinotubular bar at the commissures, but more cauded at the center with a generous mural edge. Using transmural pledgeted polypropylene sutures, the commissural ends and the sinotubular bar are suspended at the same level, with particular attention creating a suspension that best provides optimal coaptation, avoids crowding of the subcommissural triangle, and reestablishes normal semilunar appearance of each neocusp.

\section{Results}

\section{Baseline Characteristics}

Baseline characteristics are summarized in Table 1. The degree of AS and AR, length of hospital stay, and length of ICU stay did not significantly differ between the two groups ( $\mathrm{P}>0.05$ for both). Of the 38 patients reviewed, 30 patients $(75 \%)$ received autologous pericardium and $8(20 \%)$ received CorMatrix material.

\section{Aortic Stenosis}

For the entire cohort $(\mathrm{N}=40)$, the peak trans-valvular gradient significantly decreased between pre-operative and post-operative assessments $(\mathrm{P}=0.0017)$, with no significant change observed at outpatient follow-up $(\mathrm{P}=0.36)$. In the pericardium group, the peak trans-valvular gradient also significantly decreased pre- and post-operatively $(\mathrm{P}=0.012)$, with no significant change at outpatient follow-up $(\mathrm{P}=0.12)$. In the CorMatrix group, only 4 patients had AS at baseline; therefore, there was insufficient data for between-group tests (Table 2).

\section{Aortic Regurgitation}

For the entire cohort, the proportion of patients with moderate to severe regurgitation significantly decreased between pre-and post-operation $(\mathrm{P}<0.0001)$. In the pericardium group, $28(93 \%)$ had moderate to severe AR at baseline which improved to 11 (37\%) post-operatively and increased to 21 (70\%) at time of follow-up. In the biomaterial group, $8(100 \%)$ had moderate to severe AR which improved to $3(38 \%)$ post-operatively and increased to $7(88 \%)$ at time of follow-up (Table 2, Figure 2).

\section{Freedom from Re-Operation}

At five years after AVR, freedom from reoperation was significantly poorer in patients receiving CorMatrix $(12.5 \%)$ compared to patients receiving autologous pericardium $(62.5 \%)(P=0.01)$ (Figure 1). 16 patients $(42 \%)$ underwent a re-operation of the aortic valve, with 10 patients requiring re-operation due to severe or recurrent AR and 6 patients with severe, critical, or worsening AS. 6 patients underwent the Ross procedure, 2 underwent AVr with autologous pericardium, 5 underwent AVr with the Carpentier Edwards Valve, and 3 underwent AVr with the Resilia Bovine Pericardial Valve. The mean length between the initial operation and re-operation was 1390 days.

The most common reason for reoperation in the autologous pericardium group was for the repair of moderate to severe aortic regurgitation. In many of the patients, the non-augmented leaflet was prolapsing into the LV outflow, causing the AR. None of the patients receiving CorMatrix had severe AR post-operatively, however, $88 \%$ of them developed severe AR at recent-follow up (Table 2). Consequently, severe AR was the most common reason for reoperation in the CorMatrix group. Many had left and or right coronary cusps that were retracted with deformed and thickened edges. There was also lack of coaptation of the stiffened and retracted leaflets, necessitating the need for reoperation. Some cases also showed tears and delamination in the patch. 


\section{Discussion}

In the pediatric patient population, management of aortic valve disease presents a significant challenge with the overall goal of preserving the left ventricular function while also minimizing the number of reoperations required over a lifetime. Challenges involving younger patients necessitate a more complex approach due to variable anatomy, ongoing somatic growth, and available prosthesis size. ${ }^{5}$ Historically, balloon or open surgical valvotomy were used as the initial strategies to treat aortic valve disease in children and young adults, however, these techniques are associated with poor long-term outcomes and the need for additional surgical interventions within 10 years..$^{1-3}$ Aortic cusp extension valvuloplasty is an alternative that allows for better management of younger patients, with recent literature reporting favorable outcomes associated with more long-term durability. ${ }^{2-4}$ Younger patients with particular challenges involving underlying cardiac anatomy can benefit from aortic valvuloplasty, and advocates cite superior freedom from re-operations and acceptable preservation of the left ventricle function. ${ }^{1-5}$ This surgical strategy allows for annular growth until the use of a more permanent prosthesis placement is feasible.

While aortic valvuloplasty is a refined surgical method that demonstrates improvements compared to traditional balloon or open surgical valvotomy, the durability of the technique is still limited by the type of patch replacement material for cusp tissue. ${ }^{5}$ Traditional materials commonly used for valve repair include autologous pericardium, preserved homograft, bovine pericardium, and polytetrafluorethylene (PTFE). ${ }^{2-4}$ However, in both short and long-term studies, several reports cite major concerns related to lack of growth potential, calcification, thickening, and eventual degradation. ${ }^{1-5}$ Consequently, the type of material remains a major weakness in leaflet replacement surgeries, and the search for the optimal material continues to evolve as many new biological and synthetic options become available. ${ }^{4}$ Extracellular matrix (ECM), such as CorMatrix (CorMatrix Cardiovascular, Inc, Atlanta, GA), is one such material that was developed as a promising alternative because of its ability to resist calcification and serve as an interim biological scaffold that enables the propagation and regeneration of a patient's own cells to ultimately repair tissues. ${ }^{1}$ Many centers have used it for valve repair in the tricuspid, mitral, aortic, and pulmonary valve, yet the reported outcomes vary (Table 3 ).

Czub and associates reported using CorMatrix for tricuspid valve repair in the treatment of a patient with infective endocarditis. No tricuspid regurgitation or reinfection at 6 -month follow-up was reported. ${ }^{10}$ Other case studies also found favorable short-term outcomes in tricuspid and mitral valve repairs with CorMatrix, reporting successful repairs, excellent valve function, and good hemodynamic results at 3-month followup. ${ }^{11,12}$ Luk and associates, however, reported more long-term results with 10 and 12-month follow-up outcomes and found delayed post-operative infection and perforation of the MV leaflet after histological examination of explanted CorMatrix in 2 adults. ${ }^{13}$

Outcomes using CorMatrix in mitral valve repair also varied in reports studying larger patient populations, even in those with similar follow-up times. Gerdish and associates reported using CorMatrix for mitral valve repair to treat mitral regurgitation in 19 patients. Only $3 \mathrm{MV}$ reoperations were required, and the repaired valves showed good function and no evidence of calcification at median 10.9-month follow-up. ${ }^{14}$ In contrast, Kelley and associates reported the use of CorMatrix for mitral valve repair in 44 patients, with 8 patients (32\%) suffering recurrence of severe MR and 7 patients (28\%) requiring re-operation for patch failure at 12-month follow-up. ${ }^{15}$ In this case, differences in valve technique possibly contributed to the discrepancy in outcomes. In both studies, repair was used to treat mitral valve regurgitation with comparable followup times, but Gerdish and associates performed a partial or subtotal leaflet extension, while Kelley and associates performed an anterior leaflet augmentation. ${ }^{14,15}$

Interestingly, the literature describing aortic valve repairs using CorMatrix do not indicate good outcomes, irrespective of follow-up time. Hoffman and associates reported significant valve insufficiency in 5 (83\%) pediatric patients at 4 months to a 1-year follow-up, Mosala and associates demonstrated similar bad outcomes at 4-year follow-up for a pediatric patient requiring a re-operation for valve failure, severe calcification, fibrosis, and retraction. ${ }^{16,17}$ Furthermore, the studies comparing CorMatrix and autologous pericardium also 
reported no significant advantages for CorMatrix from histological, echocardiogram, and clinical data. Zaidi and associates reported the median length of time in situ for CorMatrix was approximately 2 months, significantly less than the reported time for leaflet extension for autologous pericardium. ${ }^{18}$ Nathan and associates also found that aortic valve repair with CorMatrix is associated with earlier time to reintervention compared to autologous pericardium at 5-year follow-up. ${ }^{19}$

Due to the variability of outcomes reported in the literature, the utility and advantages of CorMatrix in valve repairs remains uncertain. Nevertheless, our findings are congruent with the previous reports for the use of CorMatrix in aortic valve repair, and specifically demonstrates these outcomes in the pediatric patient population receiving repair using the cusp extension technique. Additionally, a recent study suggest that biomaterial patches are not optimal for leaflet extensions or reconstructions where the patch has a free edge. ${ }^{9}$ The retraction of the edges where the collagen reabsorbs without tissue contact can cause insufficiency, which is a possible explanation for the mechanism of failure seen in the CorMatrix extracellular membrane patch recipients.

\section{Conclusions}

Our findings did not demonstrate significant advantages of using CorMatrix during pediatric aortic valvuloplasty as compared to traditional pericardium. The biomaterial appears to be more susceptible to severe aortic regurgitation due to thickened, shortened, stiff, and retracted leaflets which caused lack of coaptation. Additional studies examining a larger patient population are warranted to further determine the long-term durability of repairs.

\section{Limitations:}

Our study has limitations inherent in all retrospective reviews. Specifically, we were limited by the small cohort size. Possible directions of future investigations should validate the durability of CorMatrix using a larger clinical experience of valve repairs.

Acknowledgments and Disclosure:

None.

\section{References}

1. d'Udekem Y. Aortic valve repair in children. Ann Cardiothorac Surg. 2013;2(1):100-104. doi:10.3978/j.issn.2225-319X.2012.11.08

2. Polimenakos AC, Sathanandam S, Elzein C, Barth MJ, Higgins RS, Ilbawi MN. Aortic cusp extension valvuloplasty with or without tricuspidization in children and adolescents: long-term results and freedom from aortic valve replacement. J Thorac Cardiovasc Surg. 2010 Apr;139(4):933-41; discussion 941. doi: 10.1016/j.jtcvs.2009.12.015.

3. David TE. Cusp repair in aortic valve procedures: advanced techniques. Tex Heart Inst J. 2013;40(5):547548.

4. Vergnat M, Asfour B, Arenz C, Suchowerskyj P, Bierbach B, Schindler Eetal. Contemporary results of aortic valve repair for congenital disease: lessons for management and staged strategy. Eur J Cardiothorac Surg 2017;52:581-7.

5. Wiggins LM, Mimic B, Issitt R, Ilic S, Bonello B, Marek J, Kostolny M. The utility of aortic valve leaflet reconstruction techniques in children and young adults. J Thorac Cardiovasc Surg. 2020 Jun;159(6):23692378. doi: $10.1016 /$ j.jtcvs.2019.09.176. 
6. Nelson JS, Heider A, Si MS, Ohye RG. Evaluation of Explanted CorMatrix Intracardiac Patches in Children With Congenital Heart Disease. Ann Thorac Surg. 2016 Oct;102(4):1329-35. doi: 10.1016/j.athoracsur.2016.03.086.

7. Polimenakos AC, Sathanandam S, Blair C, Elzein C, Roberson D, Ilbawi MN. Selective tricuspidization and aortic cusp extension valvuloplasty: outcome analysis in infants and children. Ann Thorac Surg. 2010;90(3):839-847. doi:10.1016/j.athoracsur.2010.05.052

8. Przybylski R, Pawlak S, Śliwka J, et al. Aortic cusp extension valvuloplasty: repair with an extracellular patch. Kardiochir Torakochirurgia Pol. 2015;12(4):314-317. doi:10.5114/kitp.2015.56780

9. Li Cai Haney, Hosam F. Ahmed, Alia Dani, Peter Chin, Karthik Thangappan, Nicolas Madsen, Farhan Zafar, James S. Tweddell, David L.S. Morales. Over 400 Uses of An Intestinal Submucosal Extracellular Matrix Patch in a Congenital Heart Program. Ann Thorac Surg. 2021; 0003-4975. doi.org/10.1016/j.athoracsur.2021.06.087.

10. Czub PS, Arendarczyk A, Kopala M, Budnik M, Hendzel P. Usefulness of CorMatrixbased tricuspid valve repair in the treatment of infective endocarditis. Kardiol Pol. 2018;76(2):476. doi:10.5603/KP.2018.0047

11. Sündermann SH, Rodriguez Cetina Biefer H, Emmert MY, Falk V. Use of extracellular matrix materials in patients with endocarditis. Thorac Cardiovasc Surg. 2014;62(1):76-79. doi:10.1055/s-0032-1328920

12. Wallen J, Rao V. Extensive tricuspid valve repair after endocarditis using CorMatrix extracellular matrix. Ann Thorac Surg. 2014;97(3):1048-1050. doi:10.1016/j.athoracsur.2013.05.117

13. Luk A, Rao V, Cusimano RJ, David TE, Butany J. CorMatrix Extracellular Matrix Used for Valve Repair in the Adult: Is There De Novo Valvular Tissue Seen?. Ann Thorac Surg. 2015;99(6):2205-2207. doi:10.1016/j.athoracsur.2014.08.063

14. Gerdisch MW, Shea RJ, Barron MD. Clinical experience with CorMatrix extracellular matrix in the surgical treatment of mitral valve disease. J Thorac Cardiovasc Surg. 2014;148(4):1370-1378. doi:10.1016/j.jtcvs.2013.10.055

15. Kelley TM Jr, Kashem M, Wang H, et al. Anterior Leaflet Augmentation With CorMatrix Porcine Extracellular Matrix in Twenty-Five Patients: Unexpected Patch Failures and Histologic Analysis. Ann Thorac Surg. 2017;103(1):114-120. doi:10.1016/j.athoracsur.2016.05.090

16. Hofmann M, Schmiady MO, Burkhardt BE, et al. Congenital aortic valve repair using CorMatrix ${ }^{\circledR}$ : A histologic evaluation. Xenotransplantation. 2017;24(6):10.1111/xen.12341. doi:10.1111/xen.12341

17. Mosala Nezhad Z, Baldin P, Poncelet A, El Khoury G. Calcific Degeneration of CorMatrix 4 Years After Bicuspidization of Unicuspid Aortic Valve. Ann Thorac Surg. 2017;104(6):e431-e433. doi:10.1016/j.athoracsur.2017.07.040

18. Zaidi AH, Nathan M, Emani S, et al. Preliminary experience with porcine intestinal submucosa (CorMatrix) for valve reconstruction in congenital heart disease: histologic evaluation of explanted valves. $J$ Thorac Cardiovasc Surg. 2014;148(5):2216-2225.e1. doi:10.1016/j.jtcvs.2014.02.081

19. Nathan M, Beroukhim R, Gauvreau K, et al. Aortic valve repair in the pediatric population: Midterm outcomes comparing leaflet repair using autologous pericardium versus porcine intestinal submucosa (cormatrix @). Journal of the American College of Cardiology. 2017;69(11):557. doi:10.1016/s0735-1097(17)33946-3 


\section{Tables}

Table 1. Baseline Characteristics

\begin{tabular}{llll}
\hline Characteristic & Autologous Pericardium $(\mathbf{N}=\mathbf{3 0})$ & CorMatrix $(\mathbf{N}=\mathbf{8})$ & $\mathrm{P}$ \\
Age, years & $13.63 \pm 6.5$ & $10.25 \pm 6.56$ & 0.22 \\
Weight, $\mathrm{kg}$ & $60.7(42.2,71.17)$ & $28.35(20.45,50.98)$ & 0.11 \\
Height, cm & $165(140,173)$ & $129.3(111.8,157.8)$ & 0.15 \\
Previous operation & $21(70 \%)$ & $6(75 \%)$ & 1 \\
AV anatomy & $2(1,2)$ & $2(2,3)$ & 0.66 \\
Bicuspid & $22(73 \%)$ & $7(88 \%)$ & \\
Tricuspid & $7(23 \%)$ & $1(13 \%)$ & 0.80 \\
Aortic regurgitation & & & \\
None & $0(0 \%)$ & $0(0 \%)$ & \\
Mild & $2(7 \%)$ & $0(0 \%)$ & \\
Moderate & $11(37 \%)$ & $2(25 \%)$ & \\
Severe & $17(57 \%)$ & $6(75 \%)$ & 0.58 \\
CPB time, min & $139.5(110,154.5)$ & $129.5(105.8,150.2)$ & 0.89 \\
Cross-clamp time & $112(91,126.8)$ & $107.5(86.5,128)$ & \\
\hline
\end{tabular}

$\mathrm{AV}$, aortic valve; $\mathrm{CPB}$, cardiopulmonary bypass; ${ }^{*} P<0.05$

Table 2. Recurrent Aortic Stenosis (AS) or Aortic Regurgitation (AR) After Aortic Cusp Extension Valvuloplasty

\begin{tabular}{lllll}
\hline $\begin{array}{l}\text { Variable } \\
\text { AS }\end{array}$ & $\begin{array}{l}\text { Post-Operation } \\
\text { Autologous Pericardium }(\mathbf{N}=\mathbf{3 0})\end{array}$ & $\begin{array}{l}\text { Post-Operation } \\
\text { CorMatrix }(\mathbf{N}=8)\end{array}$ & $\begin{array}{l}\text { Post-Operation } \\
\text { P }\end{array}$ & $\begin{array}{l}\text { Recent Follow Up } \\
\text { Autologous Pericard }\end{array}$ \\
Mild & $20(67 \%)$ & $5(63 \%)$ & 0.2 & $12(40 \%)$ \\
Moderate & $6(20 \%)$ & $0(0 \%)$ & & $5(17 \%)$ \\
Severe & $0(0 \%)$ & $0(0 \%)$ & & $0(0 \%)$ \\
None & $4(13 \%)$ & $3(38 \%)$ & & $13(43 \%)$ \\
AR & & & 1 & $6(20 \%)$ \\
Mild & $17(57 \%)$ & $5(63 \%)$ & & $13(43 \%)$ \\
Moderate & $10(33 \%)$ & $3(38 \%)$ & $8(27 \%)$ \\
Severe & $1(3 \%)$ & $0(0 \%)$ & $3(10 \%)$ \\
None & $2(7 \%)$ & $0(0 \%)$ & & \\
\hline
\end{tabular}

* $P<0.05$

Table 3. CorMatrix Literature Review

\begin{tabular}{lllll}
\hline Study & Valve Repaired & Patient Population & Cohort Size (n) & Follow-Up \\
\hline Gerdisch et al. & Mitral Valve & Adult & 19 & Mean 10.9 months \\
Sundermann et al. & Mitral Valve & Adult & 2 & 34 days and 3 months
\end{tabular}




\begin{tabular}{lllll}
\hline Study & Valve Repaired & Patient Population & Cohort Size (n) & Follow-Up \\
\hline Zaidi et al. & Mitral and Aortic Valve & Pediatric & 9 & MV: median 64 days AV: \\
Luk et al. & Mitral Valve & Adult & 2 & 10 and 18 months \\
Kelley et al. & Mitral Valve & Adult & 44 & 12 months \\
Wallen et. al & Tricuspid Valve & Adult & 1 & 3 months \\
Hoffman et. al & Aortic Valve & Pediatric & 6 & 119 - 441 days \\
Padalino et al. & Pulmonary and Aortic Valve & Pediatric & 22 & 23 months \\
Mosala et al. & Aortic Valve & Peadiatric & 1 & 4 years \\
Szalanski et al. & Mitral and Tricuspid Valve & Adult & 1 & 52 days \\
Czub et al. & Tricuspid Valve & Adult & 1 & 6 months \\
Nathan et al. & Aortic Valve & Pediatric & 75 & 5 years \\
\hline
\end{tabular}

\section{Figures}

\section{Figure 1.}

Title: Freedom from Reoperation by Material Received at Index Surgery

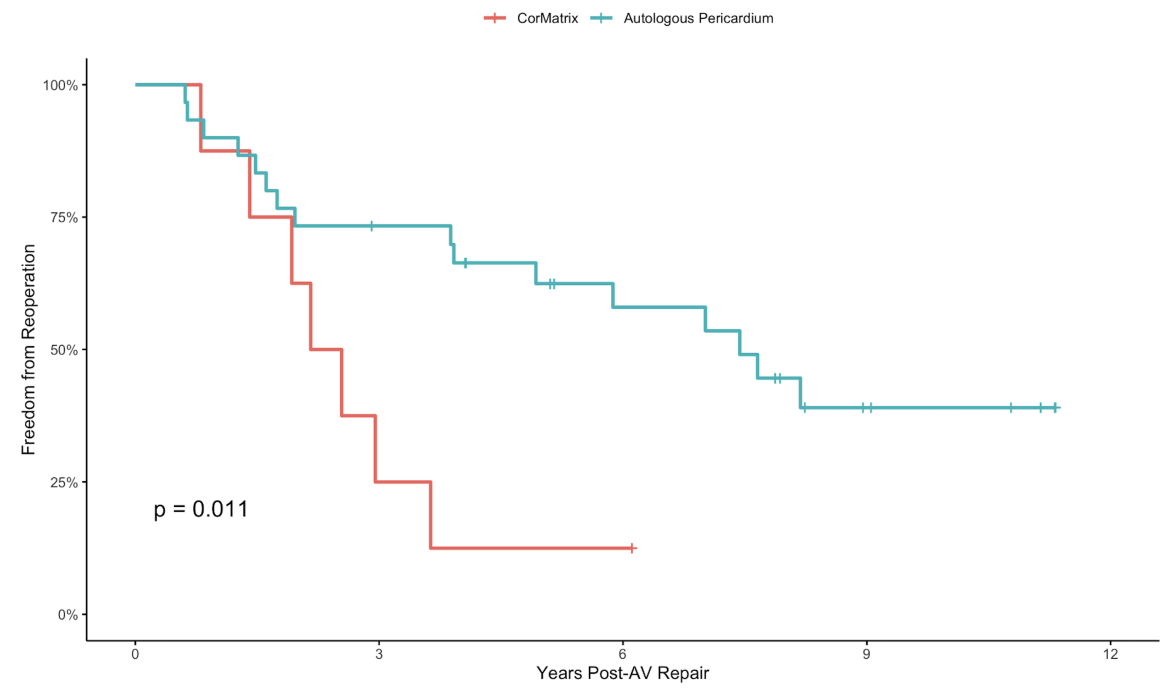

Legend: Freedom from reoperation comparison between CorMatrix and autologous pericardium showing a significantly greater freedom from reoperation for patients receiving autologous pericardium material.

\section{Figure 2.}

Title: Post-Operative Proportion of Aortic Regurgitation (AR) by Material 


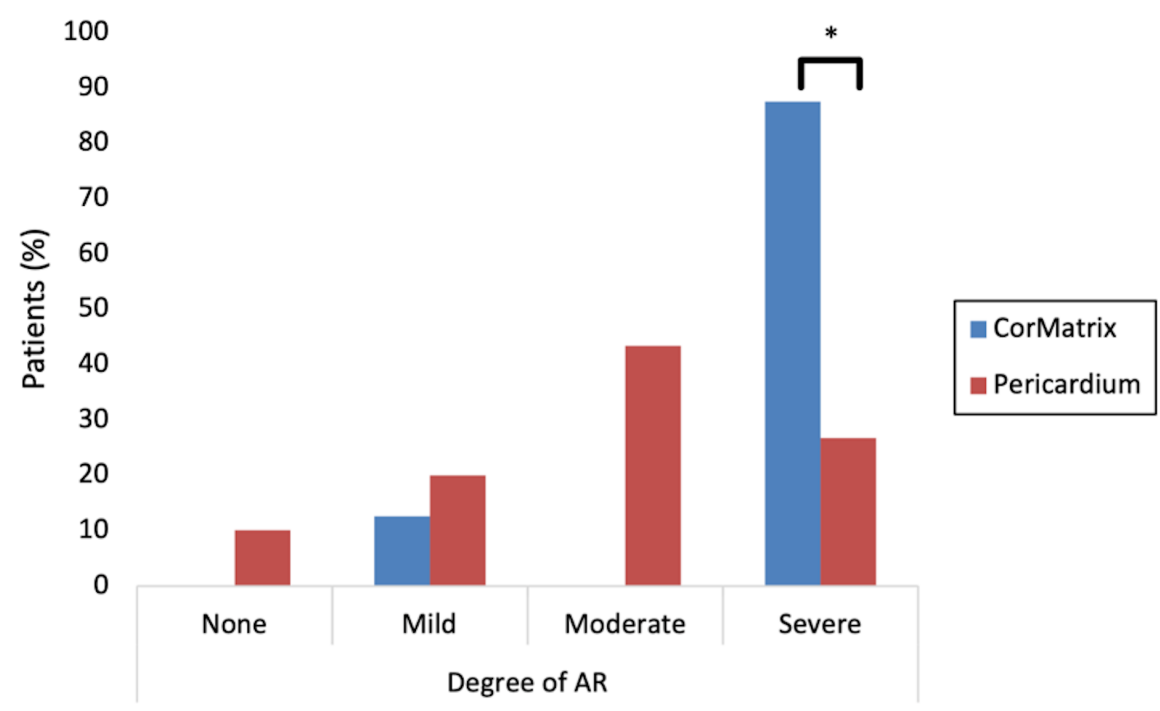

Legend: Percentage of patients with none, mild, moderate, or severe aortic regurgitation (AR) postoperatively. Shows a significantly higher percentage of CorMatrix patients with severe AR. 